\title{
Relationship between Clinical Macular Changes and Retinal Function in Age-Related Macular Degeneration
}

\author{
Peter N. Dimitrov, ${ }^{1,2}$ Liubov D. Robman, ${ }^{1}$ Mary Varsamidis, ${ }^{1}$ Khin Zaw Aung, ${ }^{1}$ \\ Galina Makeyeva, ${ }^{1}$ Lucy Busija, ${ }^{1,3}$ Algis J. Vingrys, ${ }^{2,4}$ and Robyn H. Guymer ${ }^{1,4}$
}

Purpose. The aim of this study was to investigate the relationship between clinical macular changes and retinal function in age-related macular degeneration (AMD).

\begin{abstract}
Methods. We recruited 357 participants with visual acuity of better than 20/60 in the study eye, including 64 individuals with normal fundi and 293 AMD participants classified into 12 subgroups based upon the International Classification and Grading System. Visual function in the study eye was assessed using two steady-state tests (achromatic $14 \mathrm{~Hz}$ flicker [F14Hz] and isoluminant blue color [BCT]) and two adaptation measurements (cone photo-stress recovery rate [CRR] and rod dark adaptation recovery rate $[R R R])$. The groups were compared on their average psychophysical measurements and ranked according to functional deficiency.
\end{abstract}

RESUlts. Both adaptation parameters were significantly abnormal when only hard and/or intermediate drusen were evident (compared to controls, $P<0.023$ ) and yielded considerably worse outcomes in cases with more advanced fundus changes $(P<0.001)$, but provided limited ability to discriminate between these cases (linear trend, CRR $t=0.68, P=0.50$ and RRR $t=1.76, P=0.08)$. Steady-state measurements, however, declined gradually along the entire hierarchy of fundus changes (linear trend, F14Hz $t=10.16, P<0.001$ and BCT $t=11.19, P$ $<0.001$ ) with $\mathrm{F} 14 \mathrm{~Hz}$ being able to detect significant functional

From the ${ }^{1}$ Center for Eye Research Australia, The University of Melbourne, The Royal Victorian Eye and Ear Hospital, Victoria, Australia; ${ }^{2}$ Department of Optometry and Vision Sciences, The University of Melbourne, Victoria, Australia; and ${ }^{3}$ Department of Medicine, The University of Melbourne, The Royal Melbourne Hospital, Victoria, Australia.

${ }^{4}$ These authors contributed equally as senior authors

Supported by grants from National Health and Medical Research Council (NHMRC) Project Grant 350224 (RHG, AJV), Australian Research Council Linkage Project (ARC-LP0211474), NHMRC CCRE, NHMRC practitioner fellowship (RHG), RVEEH Wagstaff Fellowship (LDR), RVEEH Research Committee and the Macular Vision Loss Support Society of Australia. Postdoctoral fellowship jointly funded by the Melbourne Centre for Epidemiology, Biostatistics, and Health Services Research and Centre for Research Excellence in Translational Neuroscience. CERA receives Operational Infrastructure Support from the Victorian Government.

Submitted for publication October 30, 2011; revised March 5, 2012; accepted June 6, 2012.

Disclosure: P.N. Dimitrov, None; L.D. Robman, None; M. Varsamidis, None; K.Z. Aung, None; G. Makeyeva, None; L. Busija, None; A.J. Vingrys, None; R.H. Guymer, None

Corresponding author: Peter N. Dimitrov, Centre for Eye Research Australia, The University of Melbourne, The Royal Victorian Eye and Ear Hospital, 32 Gisborne Street, East Melbourne, Victoria, 3002, Australia; dimitrov@unimelb.edu.au. change as early as in the intermediate drusen group, when compared to controls $(P=0.003)$.

Conclusions. Steady state thresholds (F14Hz and BCT) and clinical signs showed significant concordance across the spectrum of early AMD fundus changes. This suggests that these tests may be an effective tool for monitoring progression of AMD to supplement clinical grading. (Invest Ophthalmol Vis Sci. 2012;53:5213-5220) DOI:10.1167/iovs.11-8958

$\mathrm{D}$ iagnosis of Age-related Macular Degeneration (AMD), is currently based upon the clinical appearance of the macula. ${ }^{1}$ Fundus signs such as drusen and pigment changes represent the early stage of the disease. ${ }^{1-3}$ Grading of such signs in terms of size, number and appearance is currently the standard approach to rating the severity of early AMD and used to predict the risk of progression to late, vision threatening forms of AMD: Geographic Atrophy (GA) and/or Choroidal Neovascularisation (CNV). ${ }^{2-6}$ Using clinical grading to determine severity and risk of the progression currently underpins all clinical and epidemiological research trials in early AMD and grading scales have been developed. ${ }^{7,8}$ One scale is described in the Age-Related Eye Disease Study (AREDS) where, for example, eyes with multiple small drusen or nonextensive intermediate drusen had a lower rate of progression to late AMD (1.3\%) during 5 years follow-up period, compared to eyes with at least 1 large drusen or extensive intermediate drusen, or noncentral geographic atrophy, $18 \%{ }^{7}$ However, even in the highest risk category of the AREDS cohort, the risk of progression to late AMD was only around 50\% over 5 years. ${ }^{7,8}$ This finding suggests that fundus grading scales alone, are not specific or sensitive enough to allow accurate monitoring of early AMD changes and predict progression towards late complications. Imaging techniques such as autofluorescence or optical coherence tomography are currently being investigated as methods to enhance the diagnosis of early AMD, and ultimately to correlate structural with functional changes. ${ }^{9,10}$

In this manuscript we investigate the possibility of staging AMD by evaluating retinal function. A broad range of functional abnormalities have been well documented in the literature. ${ }^{11,12}$ In our previous work we reported on the diagnostic capacity and clinical applicability of a wide range of psychophysical tests (steady state color and temporal contrast thresholds, dark adaptation and photostress recovery). ${ }^{12}$ From 25 test-parameters, we found the following four tests, $14 \mathrm{~Hz}$ flicker and blue color thresholds, cone recovery dynamics in the photostress test and rod recovery in dark adaptation to have the best diagnostic capacity and clinical applicability in AMD. ${ }^{12}$ These tests were employed in the current study to investigate the relationship between the fundus changes and functional impairment of the retina. Such evaluation may, by giving us complementary information about the disease stage, 
enhance our ability to monitor progression, predict risk of vision loss and evaluate the effect of emerging interventions in the course of early AMD.

\section{Methods}

\section{Participants}

Individuals with early AMD in at least one eye were recruited from the Royal Victorian Eye and Ear Hospital clinics and from private ophthalmology practices. Control subjects were recruited from unrelated family members and friends of the cases. The study was conducted in accordance with the Declaration of Helsinki and written informed consent was obtained from all participants.

All participants underwent a standardized examination procedure, including assessment of best corrected visual acuity (BCVA; 4-meter $\operatorname{logMAR}$ ), clinical eye examination (slit-lamp biomicroscopy of the anterior segment and dilated ophthalmoscopy, 0.5\% tropicamide and $2.5 \%$ phenylephrine hydrochloride), digital photography using a nonmydriatic retinal camera (Canon CR6-45NM Retinal Camera; Canon, Saitama, Japan), and interview regarding history and current symptoms of eye diseases, medical history and medication. Only one eye, the study eye, underwent the psychophysical testing regimen.

The inclusion criteria for the study eye were visual acuity better than 20/60, and early AMD fundus changes of drusen and pigmentary abnormalities. ${ }^{1}$ Eyes with drusenoid pigment epithelial detachment (DPED) $)^{13,14}$ and non central GA with visual acuity fitting the inclusion criteria also were included for the psychophysical assessment. If both eyes satisfied the inclusion criteria, the eye with more advanced fundus changes was designated the study eye. However, in the cases where one eye had large drusen $(>125 \mu \mathrm{m})$ but no pigmentary abnormalities and the fellow eye had pigmentation with or without drusen, we chose the eye without pigmentation as the study eye to determine if fellow eye pigmentation had an influence on the visual function in the study eye. If the changes in both fundi were similar, then the eye with better visual acuity was chosen. In cases when visual acuity and the fundus status were the same, the right eye was assigned as the study eye. The cases with one eye eligible for psychophysical assessment and the fellow eye with late AMD also were included to determine if the visual function in the study eye is associated with the advanced condition in the fellow eye. The clinical status of the fellow eye in the AMD cohort ranged from presence of any signs of early AMD through to late stage AMD (GA and/or CNV). Control participants were of similar age to cases, and had normal fundi and a visual acuity of $20 / 20$ or better. The eye with better visual acuity was designated the study eye. If acuity was the same in both eyes then the right eye was chosen.

Exclusion criteria were significant cataract (nuclear $\geq 2.0$, cortical $\geq 0.25$, posterior subcapsular $\geq 1 \mathrm{~mm}^{2}$, according to the Wilmer grading system), ${ }^{15}$ glaucoma, heterotropia, amblyopia, color blindness, systemic hypertension uncontrolled by medication (systolic $>150$ and diastolic $>90 \mathrm{~mm} \mathrm{Hg}$ ), and presence of physical or mental impairment, neurologic or systemic diseases, and medication that might affect retinal function.

\section{Fundus Grading}

The fundus images were graded according to the International Classification and Grading System for AMD by two independent graders using "OptoMize PRO" software from Digital Healthcare Image Management System (Digital Healthcare Ltd., Cambridge, UK). ${ }^{1}$ Where disagreement occurred between the two graders, the results were adjudicated by a senior retinal specialist (RG). The data on grading agreement between the two graders has been reported previously. ${ }^{16}$

Morphologic fundus changes were classified in our study as follows. Hard drusen were defined as discrete whitish-yellow spots with diameter size $\leq 63 \mu \mathrm{m}$. Intermediate drusen were pale-yellow spots $63-$ $125 \mu \mathrm{m}$ in diameter. Soft distinct drusen were pale-yellow spots with uniform density and discrete edges larger than $125 \mu \mathrm{m}$ in diameter. Soft indistinct drusen were pale-yellow spots with decreasing density from the center outwards and fuzzy edges, larger than $125 \mu \mathrm{m}$ in diameter. Hyperpigmentation identified any sharply defined area of increased pigmentation (black or grey patches) of the RPE. Hypopigmentation identified any sharply defined area of decreased pigmentation (whitish patches) of the RPE. DPED was defined as areas of elevated central RPE caused by the confluence of soft drusen. ${ }^{13,14}$ GA was defined as sharply delineated areas of RPE hypopigmentation, larger than $175 \mu \mathrm{m}$ with visible choroidal vessels in its base. CNV included serous detachment of the sensory retina, or hemorrhagic or serous pigment epithelial detachment. Any suggestion of CNV was confirmed by fluorescein angiography. ${ }^{10,17} \mathrm{~A}$ disciform scar was defined as a well distinct patch of irregular shape and texture, white, brown, or black in color. ${ }^{1}$

\section{Psychophysical Examinations of Retinal Function}

Visual function tests included two steady-state thresholds (achromatic F14Hz and isoluminant BCT) and two adaptation measurements (CRR [cone recovery rate] and RRR [rod recovery rate]). ${ }^{12}$ All visual function tests were conducted monocularly, in the study eye, after pupil dilation of more than $7 \mathrm{~mm}$. Detailed description of the psychophysical assessment has been reported previously. ${ }^{12,18}$ In brief, stimuli for all tests were generated on a calibrated high resolution CRT monitor. ${ }^{18}$ BCT, F14Hz and the photostress (CRR) stimuli were displayed on a 30 cd. $\mathrm{m}^{-2}$ (>1100 Troland) mean background (photopic condition). A staircase ( 4 decibels $[\mathrm{dB}] \mathrm{up} / 2 \mathrm{~dB}$ down) was used to return threshold. The blue color stimulus was an equiluminous $2^{\circ}$ diameter Gaussian blob specified in $x=0.23$ and $y=0.12$ MacLeod-Boynton color space. ${ }^{19}$ To control for the effect of lens opacity on the threshold, we excluded all participants with lens opacity greater than 2.0 (Wilmer grading system). ${ }^{15}$ Flicker threshold was measured using a foveal, $2^{\circ}$ diameter Gaussian blob at $14 \mathrm{~Hz}$. The stimulus was generated with a sinusoid that had a time-averaged luminance of $30 \mathrm{~cd} \cdot \mathrm{m}^{-2}$ and a maximum Michelson contrast of 0.94 . A staircase ( $4 \mathrm{~dB}$ up $/ 2 \mathrm{~dB}$ down) was used to return threshold.

CRR measurements were acquired using the photostress recovery test described previously. ${ }^{12,19}$ In short, the average of four pre-bleach threshold estimates for a foveal $2^{\circ}$ spot flickering at $5 \mathrm{~Hz}$ was determined in the study eye. The eye then was exposed to a light source $\left(12 \times 10^{6} \mathrm{~cd} \cdot \mathrm{m}^{-2}\right)$ that bleached $>95 \%$ of photopigment and recovery was tracked immediately after the light was turned off. Recovery time was recorded at five suprathreshold contrast levels (prebleach threshold $[\mathrm{PT}] \times 3.5, \times 3.0, \times 2.5, \times 2.0, \times 1.5) .{ }^{12}$ The dynamics of the recovery were modelled using a single exponential decay. ${ }^{18}$

RRR measurements were acquired on dark adaptation testing with a $2^{\circ}$ stimulus at $3.5^{\circ}$ inferior retina along the vertical meridian. ${ }^{2,18}$ The $3.5^{\circ}$ eccentric location was chosen because rods at this eccentricity were found to be most vulnerable in AMD. ${ }^{20,21}$ Furthermore, we have shown previously that measuring dark adaptation in this location had good diagnostic capacity for early stages of AMD, ${ }^{12}$ consistent with other reports. ${ }^{22}$ Therefore, we adopted this parameter to evaluate the rod function in participants with various fundus grades in the current study. Dark adaptation was monitored after bleaching 30\% of photopigment. ${ }^{12}$ Rod recovery was modelled as detailed previously using a single exponential decay $\left(\log _{10} \cdot \mathrm{min}^{-1}\right){ }^{18}$

\section{Statistical Analyses}

Participants were grouped according to AMD-related macular changes in both eyes. These groups were arranged in a hierarchy of fundus features with increasing risk of progression to late stage of the disease. ${ }^{2-6}$

Examining the distribution of psychophysical measurements (Kolmogorov-Smirnov test) revealed significant positive skewness for all four measurements. After $\log$ transformation, the data yielded a normal distribution with acceptable skewness (ranging from 0.830.45) and kurtosis (ranging from 0.94-0.68). Thus, all group 
TABLE. Distribution of the Study Cohort $(n=357)$ along Groups Corresponding to Clinical Signs

\begin{tabular}{|c|c|c|c|c|c|c|c|}
\hline \multirow[b]{2}{*}{ Group } & \multicolumn{2}{|c|}{ Fundus Status } & \multirow[b]{2}{*}{$\boldsymbol{N}$} & \multicolumn{2}{|c|}{ Age } & \multicolumn{2}{|c|}{ Visual Acuity } \\
\hline & Study Eye* & Fellow Eye & & Mean & $\pm \mathbf{S D}$ & Mean & $\pm \mathbf{S D}$ \\
\hline 1 & No fundus pathology & No fundus pathology & 64 & 69.16 & 11.35 & -0.07 & 0.07 \\
\hline 2 & Hard drusen only $(\leq 63 \mu \mathrm{m})$ & Same or no fundus changes & 59 & 72.09 & 11.3 & -0.07 & 0.07 \\
\hline 3 & $\begin{array}{l}\text { Intermediate drusen }>63 \text { to } \leq 124 \mu \mathrm{m} \\
\text { in size }\end{array}$ & $\begin{array}{l}\text { Same as study eye or as in the group } \\
\text { above }\end{array}$ & 12 & 75.06 & 9.07 & -0.02 & 0.08 \\
\hline 4 & $\begin{array}{l}\text { Soft distinct drusen }(\geq 125 \mu \mathrm{m}) \text { and no } \\
\text { pigmentary changes }\end{array}$ & $\begin{array}{l}\text { Same as study eye or as in the groups } \\
\text { above }\end{array}$ & 24 & 61.97 & 10.52 & -0.02 & 0.11 \\
\hline 5 & $\begin{array}{l}\text { Soft indistinct drusen }(\geq 125 \mu \mathrm{m}) \text { with/ } \\
\text { without soft distinct drusen, no } \\
\text { pigmentary changes }\end{array}$ & $\begin{array}{l}\text { Same as study eye or as in the groups } \\
\text { above }\end{array}$ & 26 & 72.04 & 9.59 & 0.02 & 0.13 \\
\hline 6 & $\begin{array}{l}\text { Any soft drusen }(\geq 125 \mu \mathrm{m}) \text {; no } \\
\quad \text { pigmentary changes } \geq 125 \mu \mathrm{m}\end{array}$ & $\begin{array}{l}\text { Any pigmentary abnormalities } \geq 125 \mu \mathrm{m} \\
\text { with/without any drusen }\end{array}$ & 12 & 73.33 & 10.22 & 0.01 & 0.12 \\
\hline 7 & $\begin{array}{l}\text { Hyperpigmentation } \geq 125 \mu \mathrm{m} \text { and any } \\
\quad \text { drusen }>63 \mu \mathrm{m}\end{array}$ & $\begin{array}{l}\text { Same as study eye or }>63 \mu \mathrm{m} \text { drusen } \\
\text { only }\end{array}$ & 20 & 69.04 & 14.39 & 0.02 & 0.15 \\
\hline 8 & $\begin{array}{l}\text { Hypopigmentation } \geq 125 \mu \mathrm{m} \text { and any } \\
\quad \text { drusen }>63 \mu \mathrm{m}\end{array}$ & $\begin{array}{l}\text { Same as study eye or }>63 \mu \mathrm{m} \text { drusen } \\
\text { only }\end{array}$ & 27 & 73.76 & 9.55 & 0.09 & 0.16 \\
\hline 9 & $\begin{array}{l}\text { Drusenoid pigment epithelial } \\
\text { detachment }\end{array}$ & $\begin{array}{l}\text { Same as study eye or as in the groups } \\
\text { above }\end{array}$ & 12 & 74 & 6.09 & 0.11 & 0.18 \\
\hline 10 & $\begin{array}{l}\text { Noncentral geographic atrophy outside } \\
1000 \mu \mathrm{m} \text { central circle and }>175 \mu \mathrm{m} \\
\text { in size }\end{array}$ & $\begin{array}{l}\text { Same as study eye or as in the groups } \\
\text { above }\end{array}$ & 14 & 76.05 & 9.94 & 0.14 & 0.19 \\
\hline 11 & $\begin{array}{l}\text { Any soft drusen with/without any } \\
\text { pigmentary abnormality }\end{array}$ & $\begin{array}{l}\text { Geographic atrophy }>175 \mu \mathrm{m} \text { in size, } \\
\text { within } 1000 \mu \mathrm{m} \text { central circle }\end{array}$ & 17 & 73.8 & 11.51 & 0.08 & 0.17 \\
\hline 12 & $\begin{array}{l}\text { Any soft drusen with/without any } \\
\text { pigmentary abnormality }\end{array}$ & Active neovascular AMD & 14 & 73.86 & 9.58 & 0.04 & 0.15 \\
\hline 13 & $\begin{array}{l}\text { Any soft drusen with/without any } \\
\text { pigmentary abnormality }\end{array}$ & Disciform scar & 56 & 76.37 & 8.98 & 0.1 & 0.16 \\
\hline
\end{tabular}
groups.

${ }^{*}$ Fundus status in the study eye in groups 3 to 10 may or may not include fundus features corresponding to the changes of the preceding

comparisons were conducted using parametric tests of transformed values (univariate analysis of covariance [ANCOVA] with age and sex as covariates, and post hoc comparisons with Bonferroni correction; SPSS, PASW Statistics 18). All $P$ values reported in this manuscript were adjusted for multiple comparisons (Bonferroni correction). Reproducibility of the psychophysical measurements has been reported previously. ${ }^{12}$

\section{Results}

Participants $(n=357)$ were classified into 13 study groups according to the morphologic fundus changes (see Table). There were 64 control subjects with no fundus abnormalities in either eye (group 1), 59 participants with hard drusen only in their study eye (fellow eye had either hard drusen only or no fundus changes, group 2), 12 with intermediate drusen in their study eye as their worst feature (status of the fellow eye was the same or there were hard drusen only, group 3) and 121 had soft drusen $(>125 \mu \mathrm{m}$ in size) with or without pigment changes in their study eye (status of the fellow eye was the same or better, groups 4-9). There also were 14 cases of noncentral GA in the study eye (fellow eye had noncentral GA or early AMD changes, group 10) and 87 cases of late AMD in the non-study eye (the study eye had early AMD changes, groups 11-13).

All participants in the control group had normal BCVA (LogMAR 0.0 [20/20] and better). LogMAR BCVA in the study eye of participants with AMD changes ranged from -0.22 to $0.48(20 / 10[-4]-20 / 60[+1])$ and $93 \%$ of individuals with AMD had BCVA within the 95\% confidence interval (CI) of age matched normal values ${ }^{23}$ (see Table). BCVA in the fellow eye of individuals with AMD ranged from -0.2 (20/10) to light perception.

\section{Visual Function in Cases with Drusen without Pigment Change}

The psychophysical outcomes in study groups with various drusen characteristics but no pigmentary abnormalities (groups 2-5, see Table) were compared to those of controls (group 1) after adjusting for age and sex. As shown in Figures 1A-1D, functional abnormalities increased consistently with increasing drusen grade. The cases with soft distinct or indistinct drusen $(>125 \mu \mathrm{m}$, groups 4 and 5) had the worst outcomes across all tests (Fig. 1). These two groups were significantly different when compared to controls (all tests, $P$ value ranged between 0.011 and $<0.001$, Fig. 1 ). The average measurements for dynamic and static tests in these two groups did not differ significantly from each other $(P$ value range $1.00-$ 0.49 , Fig. 1). The group with soft indistinct drusen (group 5) was significantly different from the group with hard drusen (group 2) on all four measurements (all tests, $P<0.001$ ). The group with distinct drusen (group 4 ) also differed significantly from hard drusen group (group 2) on all but the blue color threshold (F14Hz $P=0.001$, BCT $P=0.059$, CRR and RRR $P<$ 0.001 ; Fig. 1).

The intermediate drusen group (group 3, see Table) also revealed significant functional abnormality when compared to controls (Fig. 1). This was evident on all tests except for BCT (F14Hz $P=0.003$, BCT $P=1.00$, CRR $P=0.020$, and RRR $P=$ 0.004 ; Fig. 1). F14Hz also was capable of differentiating the intermediate drusen group from the group with hard drusen 

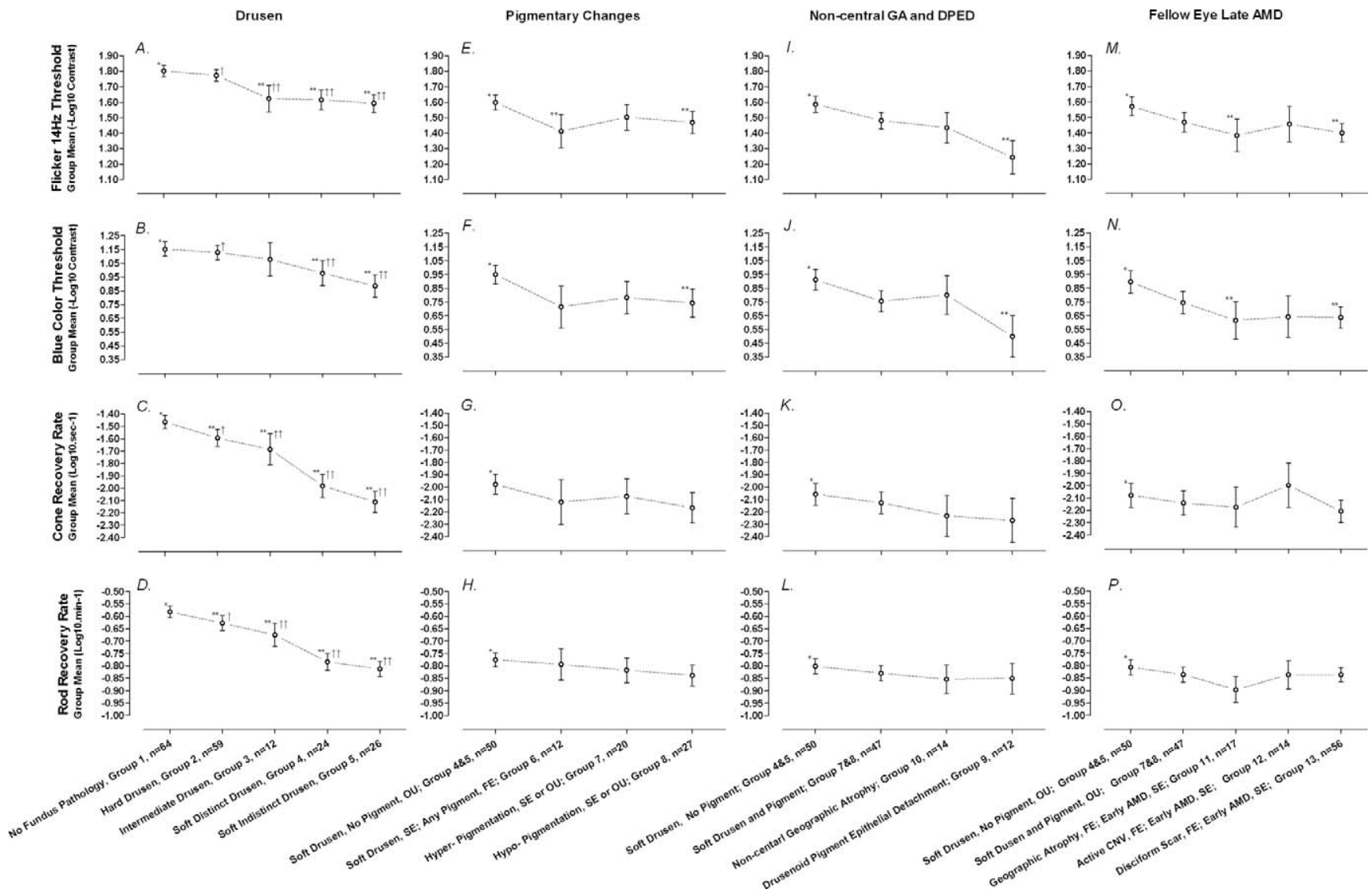

Figure 1. (A, B) Means ( \pm SEM, adjusted for age and sex) of visual function outcomes in groups with various drusen characteristics in their study eye. $(\mathbf{C}-\mathbf{H})$ In groups with macular pigmentary changes in study eye $(S E)$ or in fellow eye $(F E)$ and in the group with soft drusen in both eyes. (I-L) In groups with noncentral geographic atrophy, drusenoid pigment epithelial detachment, soft drusen with pigmentary abnormalities, and soft drusen without pigmentary abnormalities in study eye. (M-P) In AMD subjects with early AMD changes in their study eye and late AMD changes in their fellow eye, and cases with early AMD in both eyes. *First reference group. †Second reference group. ${ }^{* *}$ Significantly different groups. ††Significantly different groups. For consistent visual representation of the functional decline on all four tests, the steady state measurements (F14Hz and BCT) are reversed (-log) so that higher values represent better outcomes as in the adaptation test results (CRR and RRR).

only (group 2 vs. group $3, P=0.019$ ). When comparing the hard drusen only group (group 2) with the control group (group 1) we found significant functional abnormalities on both adaptation tests (CRR $P=0.015$ and RRR $P=0.023$ ) but not for steady state thresholds $(\mathrm{F} 14 \mathrm{~Hz}$ and BCT $P=1.00)$.

\section{Visual Function in Cases with Pigmentary Abnormalities}

To investigate the variation of retinal function in cases with macular pigmentary changes the following groups were compared after adjusting for age and sex: Group 7 (study eye with soft drusen and hyperpigmentation, and fellow eye same or better, see Table), group 8 (study eye with soft drusen, hyper- and hypo-pigmentation, and fellow eye same or better), group 6 (study eye with soft drusen and no pigmentary abnormalities, and fellow eye with soft drusen, hyper- and hypo-pigmentation), and participants with soft drusen and no pigment changes in both eyes (combined groups 4 and 5, Figs. $1 \mathrm{E}-1 \mathrm{H})$

We found no significant differences between groups with pigmentary changes (group 6 vs. group 7 vs. group 8 , all tests $P$ $=1.00$, Fig. 1). However, when these groups were compared to the groups without pigmentary changes (only soft drusen in both eyes, combined groups 4 and 5, Fig. 1) it was evident that the presence of hypo-pigmentary abnormalities (group 8) was associated with worse performance on both steady state thresholds (group 8 vs. combined groups 4 and 5, F14Hz $P=$ 0.043 and BCT $P=0.013$ ). Furthermore, the presence of hypopigmentation in the fellow eye, but not in the study eye (group 6), resulted in significantly worse values for $\mathrm{F} 14 \mathrm{~Hz}$ threshold (group 6 vs. combined groups 4 and 5, $P=0.024$, Fig. 1). The BCT in this group, although not significantly different from the reference group, also showed a trend toward worse function (group 6 vs. combined groups 4 and 5, $P=$ 0.066). Results for steady state measurements obtained from the group with hyper-pigmentary changes, without hypopigmentation (group 7) were not significantly different from the results of cases with soft drusen only, (group 7 vs. combined groups 4 and 5 , F14Hz $P=0.54$, BCT $P=0.16$, Fig. 1).

Both adaptation measurements were not significantly different between groups with pigmentary changes and cases who had soft drusen only (groups 6-8, and combined groups 4 and 5, $P$ value ranged from $1.00-0.121$, Fig. 1).

\section{Retinal Function in Cases with Advanced Fundus Changes (DPED and Noncentral GA)}

There were 14 participants with GA in their study eye, whose lesion was located outside the fovea, within a ring surrounding the fovea between 500 and $3000 \mu \mathrm{m}$ radius borders. The total area of GA lesions ranged from 175 to $3000 \mu \mathrm{m}$. Average visual acuity in the study eye of this group was 0.17 LogMAR, ranging from -0.08 to $0.46(20 / 20+3-20 / 60+2)$. The average functional 
measurements for all tests of these cases (group 10) were not different from either the cases with soft drusen without pigmentary changes or cases with soft drusen and pigmentary changes (group 10 vs. combined groups 4 and 5 and versus combined groups 7 and 8 , all tests $P$ value ranged from 1.000.097, Figs. 1I-1L). On the other hand, eyes with DPED had significantly worse measurements on both steady state thresholds when compared to the soft drusen cases (group 9 vs. combined groups 5 and $6, \mathrm{~F} 14 \mathrm{~Hz}$ and BCT $P<0.001$, Fig. 1) or cases with pigmentary changes (group 9 vs. combined groups 7 and 8 , F14Hz $P=0.001$, BCT $P=0.034$, Fig. 1).

\section{Retinal Function in Eyes with Unilateral Early AMD, Where Fellow Eyes Had Late AMD}

Cases with early AMD in the study eye, where the fellow eye had late AMD, did not differentiate between each other on any of the psychophysical measurements (group 11 vs. 12 vs. 13 , all tests $P$ value ranged from 1.00-0.615, Fig. 1). However the cases with GA (group 11) and disciform scar (group 13) in the fellow eye performed significantly worse on both steady state measurements when compared to cases that had only soft drusen in both eyes (group 11 vs. combined groups 4 and 5 , F14Hz $P=0.042$, BCT $P=0.011$; group 13 vs. combined groups 4 and 5 , F1 $4 \mathrm{~Hz} P=0.002$, BCT $P<0.001$ ). These two groups did not differ significantly from the group with pigmentary abnormalities (combined groups 7 and 8) on both steady state tests $(\mathrm{F} 14 \mathrm{~Hz}$ and BCT $P$ values ranged from $1.00-$ 0.931). The functional capacity of the cases with active CNV (group 12) was affected less than the cases with GA or disciform scar as they did not differ significantly either from the bilateral drusen group or from the cases with pigmentary changes on both steady state tests (group 12 vs. combined groups 4 and 5, and versus combined groups 7 and $8, F 14 \mathrm{~Hz}$ and BCT $P$ values ranged from 1.00-0.06).

Outcomes of both dynamic tests, although significantly worse than for the control group $(P<0.001)$, were not able to differentiate between either of the unilateral late AMD groups or the bilateral early AMD group ( $P$ value ranged from 1.000.052).

\section{Hierarchy of Early AMD Changes Based upon Visual Function Outcomes}

We used the results of psychophysical tests to determine a severity scale of functional loss and compared this to the clinical fundus changes. Figure 2 shows distribution of the study groups (see Table) sorted according to the means for four psychophysical measurements. The distribution of the steady state results revealed significant functional decline (linear trend, adjusted for age and sex, F14Hz $t=10.16$ and BCT $t=$ $11.19, P<0.001$, degrees of freedom [df] = 342) that conformed reasonably closely with clinical severity scales ${ }^{2-6}$ (Figs. 2A, 2B). This indicated that both measurements deteriorated consistently as clinical grading increased in severity. Of note, the DPED in the study eye yielded the worst thresholds (F14Hz and BCT) among all groups (Figs. 2A, 2B).

Adaptation tests (CRR and RRR), on the other hand, exhibited a rapid decrease in functional outcomes at the earliest stages of the disease, with decline being seen even when only hard drusen were evident. Figures 2C and 2D show significant reduction in recovery rates for rods and cones along the groups with hard and intermediate drusen (linear trend, adjusted for age and sex, groups $1-4$, CRR $t=-10.18$ and RRR $t$ $=-10.68, P<0.001, \mathrm{df}=158)$. However once the fundus changes included soft drusen or worse (groups 4-13, Figs. 2C, 2D) the adaptation results remained at a consistent low level of function and did not differ significantly between the groups with advanced changes (linear trend, adjusted for age and sex, groups 4-13, CRR $t=0.68, P=0.50$ and RRR $t=1.76, P=0.08$, $\mathrm{df}=209)$.

\section{Discussion}

Judicious selection of retinal functional tests could provide a valuable tool, in addition to the funduscopic examination, when monitoring AMD and its progression over time. Previous reports on the relationship between fundus appearance and retinal function have been based on small numbers of subjects, a narrow range of clinical fundus changes, and a limited number of psychophysical tests. ${ }^{24-27}$ In our current study, we examined a large number of participants $(n=357)$ over the broad range of fundus changes associated with AMD $(n=293)$ and normal control subjects without any fundus abnormalities $(n=64)$. The four psychophysical measurements explored in our study used adaptation (rod and cone mediated) and steady state (cone mediated) visual function measurements. ${ }^{12}$ We chose these parameters from our previously reported suite of tests, due to their good diagnostic capacity and practical applicability, as the ultimate aim is to be able to use these tests in the clinic and in trial settings. ${ }^{12}$ Although both dynamic tests (CRR and RRR) are challenging to apply, they gave the best diagnostic capacity when visual function in the entire AMD cohort was assessed ${ }^{12}$ and, as is evident in the current study, they are sensitive enough to detect the very early AMD changes (Figs. 1C, 1D). Two steady state tests (BCT and F14Hz) had both good diagnostic capacity and effective clinical applicability, ${ }^{12}$ and in the current analyses were shown to be effective in tracking AMD severity (Fig. 2). Although other steady state parameters, such as dark adapted absolute thresholds (rod and cone), provide a useful correlate with the grade of AMD (data not shown), their difficult implementation makes these tests less desirable ${ }^{12}$; therefore, in this study the selection was limited to BCT and F14Hz.

The large number of AMD participants with a wide range of fundus changes allowed us to divide our cohort into a substantial number of study groups (13 groups, see Table), ${ }^{1}$ thus enabling us to correlate the broad variation of the fundus changes with the outcomes of the selected functional assays. Although there were small numbers in some groups, the smallest having 12 cases (see Table), we were able to identify significant differences even in comparisons of functional outcomes involving small groups (Fig. 1). The order of the study groups in the Table is based on clinical and epidemiologic evidence for the risk of AMD progression. As such, our ordinal severity scale, represented by the group sequence, neither implies equal spacing between the groups, nor is meant to be a representation of severity order of the disease structure-functional changes. Subsequently, when clinical groups were sorted by functional outcomes, we found that each test depicted a specific rank-order of functional impairment across the clinical groups (Fig. 2). Outcomes of all functional tests exhibited significant linear trends with increasing severity of fundus grading $(P<0.001$, Fig. 2). Performance on dynamic testing of adaptation, however, was abnormal very early on in the clinical severity ranking, with significantly poorer function being seen as early as in eyes with only hard drusen evident, becoming progressively worse with intermediate then soft drusen (groups 1-4; Figs. 2C, 2D; linear trend, $P<0.001$ ) and then plateauing at a low level of function that remained stable despite more severe clinical stages (groups 4-13; Figs. 2C, 2D; linear trend, CRR $P=0.50$ and RRR $P=0.08)$. Steady state tests $(\mathrm{F} 14 \mathrm{~Hz}$ and $\mathrm{BCT})$, on the other hand, showed a gradual decline in visual function with increasing severity of fundus grade (Figs. 2A, 2B). This decline 
A.

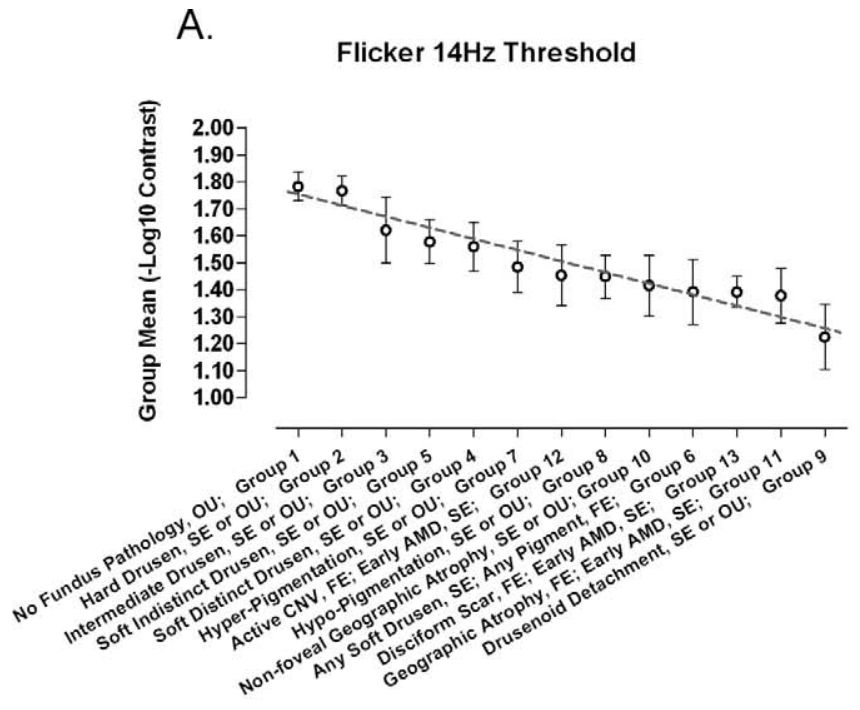

C.

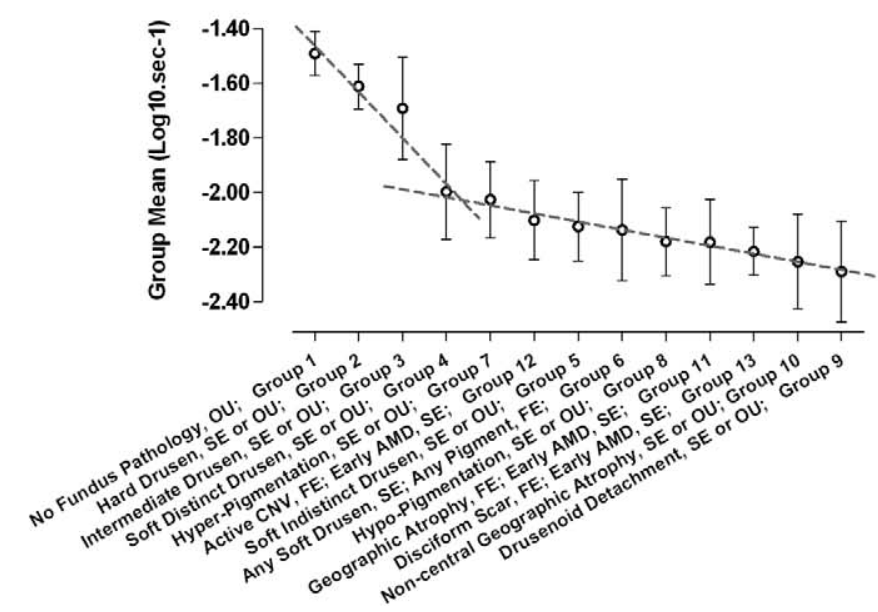

B.

Blue Colour Threshold

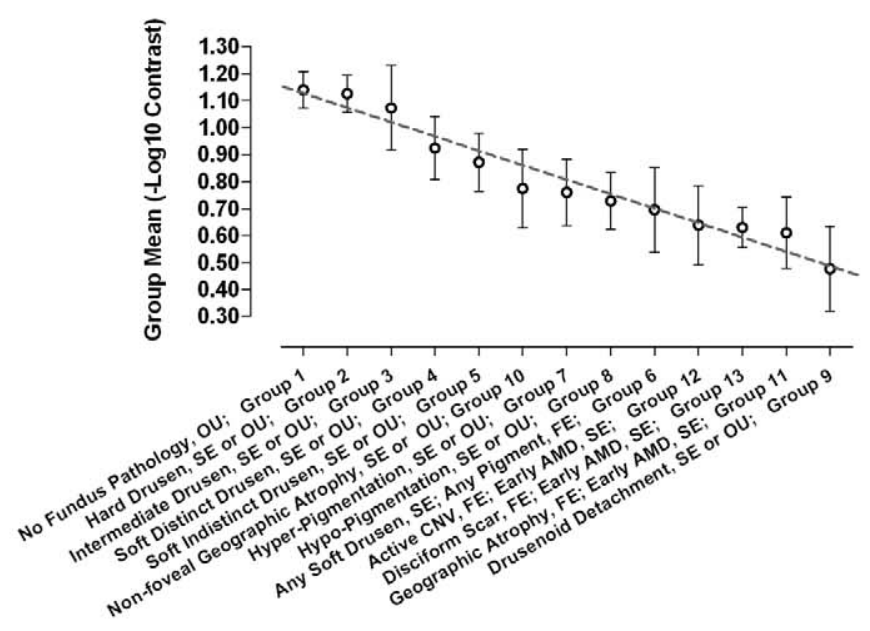

D.

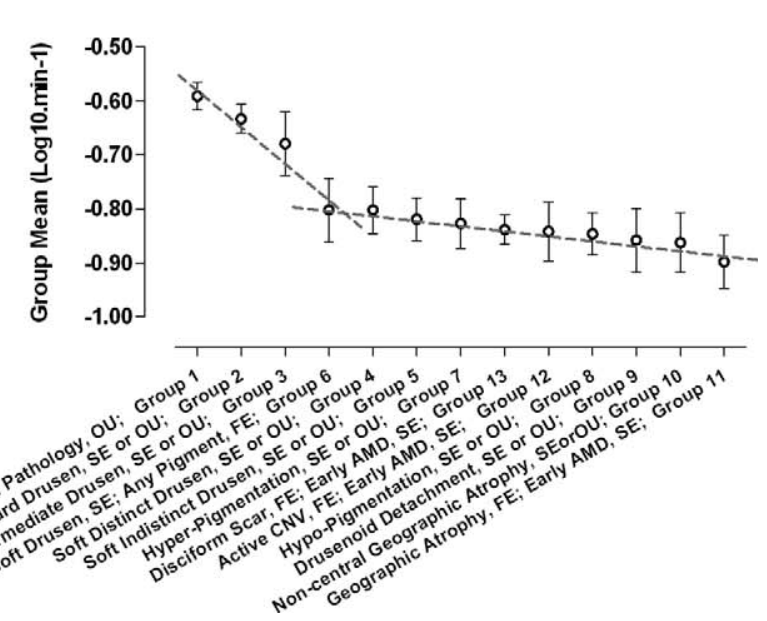

Figure 2. AMD clinical grading hierarchy based upon functional outcomes. Ranking has been determined from group averages of the psychophysical measurements ( \pm SEM, adjusted for age and sex). Groups are classified according to fundus status of a subject's study eye ( $S E$ ), fellow eye $(F E)$, or both eyes $(O U)$. For consistent visual representation of the functional decline on all four tests, the steady state measurements (F14Hz and BCT) are reversed (-log) so that higher values represent better outcomes as in the adaptation test results (CRR and RRR).

followed a linear trend $(\mathrm{F} 14 \mathrm{~Hz}$ and BCT $P<0.001)$ and is reasonably consistent with our current clinical understanding of worsening signs of AMD. ${ }^{2-6}$ Worsening function was evident with increasing size of drusen and when pigmentary abnormalities were added. However, test results for some groups were not in the order of what might have been considered increasing clinical severity. ${ }^{2-6}$ For example, when looking at the results of steady state tests, group 12 (fellow eye has CNV) returns better outcomes than might have been predicted from the hierarchy of implied risk of progression based on clinical assessment. Whereas the worst AMD-related fundus changes in the study eye to produce the greatest loss of function was the group with DPED (group 9, Fig. 2). The differences between clinical severity and various outcomes of functional tests imply that each testing modality may provide different information about the underlying pathologic processes. ${ }^{28}$ These findings suggested that psychophysical assessment of visual function in AMD patients offers a valuable addition to the clinical estimation of the severity of fundus changes.
Hard drusen are very common in people over the age of 50 years and are not considered part of a diagnosis of AMD by clinicians. In our study, we had 59 cases with hard drusen as the only fundus change. We compared visual function in this group to 64 control cases with no fundus changes in either eye. Comparison of the steady state results revealed a nonsignificant trend toward worsening in the hard drusen group (Fig. 1, F14Hz and BCT, $P=1.00$ ). However, both adaptation tests showed significantly abnormal outcomes in the hard drusen group when compared to controls (CRR $P=0.015$ and RRR $P=$ 0.023). This finding lends weight to the idea that hard drusen signify the pathologic process of the early stage of AMD. The intermediate drusen group not only had significant abnormalities on both adaptation tests (groups 1 vs. 3, CRR $P=0.020$ and RRR $P=0.004$ ) but also showed reduced $14 \mathrm{~Hz}$ flicker sensitivity (groups 1 vs. $3, \mathrm{~F} 14 \mathrm{~Hz} P=0.003$ ). However, wide variations in the outcomes in this group (group 3, Figs. 1A1D), where some participants returned normal results while others clearly were abnormal, might be giving us new insight into the pathology of early disease. The functional test results 
might be differentiating patients who are most likely to progress from those with less risk. To determine if the differing response seen in this early stage is indicative of future course of the disease, a prospective study would be needed where individuals with early fundus changes are monitored over time using these tests.

When the AMD status (early or late) in the fellow eye was taken into consideration we found that the presence of late AMD in the fellow eye was associated significantly with worse functional results in the study eye when compared to cases of bilateral early AMD. The presence of GA and longstanding CNV compromised the visual function in the fellow eye to a significant extent (Fig. 1). However, the functional capacity of the eyes where the fellow eye recently had acquired CNV complications did not differ significantly from the cases with bilateral early AMD (Fig. 1). Since the clinical grades of the study eyes in longstanding and new CNV cases were the same, the difference in the functional capacity between these two groups implies that the degenerative process due to the longstanding complications in one eye might influence the disease process in the fellow eye.

Our findings indicate that adaptational parameters become abnormal early, reach a point of very poor function, and then remain at this lower level. Dark adaptation is known to be abnormal early in $\mathrm{AMD}^{11,12}$ and is thought to occur due to a thickened Bruch's membrane (BM), ${ }^{29,30}$ which has a detrimental effect on the visual cycle in rods and cones. ${ }^{31,32}$ Our results suggest that even at the stage when only hard drusen are present, before we can identify obvious clinical signs of early AMD, changes in BM already are profound enough to compromise the visual adaptation significantly (Fig. 1), reaching a point of maximal dysfunction with appearance of large drusen (Fig. 2), beyond which there is no further worsening. Therefore, the adaptation tests would be of most benefit to define the earliest possible change in function and potentially could have a role in screening genetically at risk individuals before clinical signs were apparent. These tests, however, would be of less use in monitoring progression over time once significant early AMD had developed as the tests' results remained consistently poor.

On the other hand, the steady state tests declined along the continuum of the clinical grades starting with minor retinal changes and deteriorating as clinical disease severity increased. The results appear to be independent of the adaptation process. Although both steady state tests showed good correlation with clinical fundus changes, the $14 \mathrm{~Hz}$ flicker yielded higher sensitivity for differentiating cases with intermediate drusen from the controls (Fig. 1) and the cases with bilateral drusen (no pigment changes) from the cases with unilateral pigment change (Fig. 1). A flickering stimulus increases the metabolic demand of retinal tissues, producing a compensatory retinal vasodilatation, ${ }^{33}$ increased local blood flow, ${ }^{34}$ and altered oxygen tension. ${ }^{35}$ The abnormal functional outcomes in our study suggest that this compensatory mechanism is compromised in eyes with early AMD changes, including intermediate drusen, possibly due to either abnormalities of the choriocapillary vasculature, ${ }^{36-40}$ disturbance of RPE/Bruch's complex, ${ }^{29,30,41}$ or because the flicker stimulus targets post-receptoral (inner retina) impairment ${ }^{19,42,43}$ and ischemic changes in this retinal region. ${ }^{44}$ Furthermore, our results tend to indicate that this characteristic changes progressively across the entire spectrum of disease severity. Therefore, this test would have a good potential to monitor disease progression and detect possible stabilization or reversal of the process with new treatment.

Our study has given us a unique resource with an opportunity to divide the disease into various clinical stages and advance our thinking as to potential clinically applicable functional markers of early AMD with applications for monitoring progression, predicting vision loss, and assessing the success of novel interventions.

\section{References}

1. Bird AC, Bressler NM, Bressler SB, et al. An international classification and grading system for age-related maculopathy and age-related macular degeneration. The International ARM Epidemiological Study Group. Surv Ophthalmol. 1995;39: 367-374.

2. Davis MD, Gagnon RE, Lee LY, et al. The Age-Related Eye Disease Study severity scale for age-related macular degeneration: AREDS Report No. 17. Arch Ophthalmol. 2005;123: 1484-1498.

3. van Leeuwen R, Klaver CC, Vingerling JR, Hofman A, de Jong PT. The risk and natural course of age-related maculopathy: follow-up at $61 / 2$ years in the Rotterdam study. Arch Ophthalmol. 2003;121:519-526.

4. Klaver CC, Assink JJ, van Leeuwen R, et al. Incidence and progression rates of age-related maculopathy: the Rotterdam Study. Invest Ophthalmol Vis Sci. 2001;42:2237-2241.

5. Wang JJ, Foran S, Smith W, Mitchell P. Risk of age-related macular degeneration in eyes with macular drusen or hyperpigmentation: the Blue Mountains Eye Study cohort. Arch Ophthalmol. 2003;121:658-663.

6. Klein R, Klein BE, Tomany SC, Meuer SM, Huang GH. Ten-year incidence and progression of age-related maculopathy: the Beaver Dam eye study. Ophthalmology. 2002;109:1767-1779.

7. Age-Related Eye Disease Study Research Group. A randomized, placebo-controlled, clinical trial of high-dose supplementation with vitamins $\mathrm{C}$ and $\mathrm{E}$, beta carotene, and zinc for age-related macular degeneration and vision loss: AREDS report no. 8. Arch Ophthalmol. 2001;119:1417-1436.

8. Ferris FL, Davis MD, Clemons TE, et al. A simplified severity scale for age-related macular degeneration: AREDS Report No. 18. Arch Ophthalmol. 2005;123:1570-1574.

9. Stanga PE, Bird AC. Optical coherence tomography (OCT): principles of operation, technology, indications in vitreoretinal imaging and interpretation of results. Int Ophthalmol. 2001; 23:191-197.

10. Lois N, Owens SL, Coco R, Hopkins J, Fitzke FW, Bird AC. Fundus autofluorescence in patients with age-related macular degeneration and high risk of visual loss. Am J Ophthalmol. 2002;133:341-349.

11. Neelam K, Nolan J, Chakravarthy U, Beatty S. Psychophysical function in age-related maculopathy. Surv Ophthalmol. 2009; 54:167-210.

12. Dimitrov PN, Robman LD, Varsamidis M, et al. Visual function tests as potential biomarkers in age-related macular degeneration. Invest Ophthalmol Vis Sci. 2011;51:9457-9469.

13. Casswell AG, Kohen D, Bird AC. Retinal pigment epithelial detachments in the elderly: classification and outcome. $\mathrm{Br} \mathrm{J}$ Ophthalmol. 1985;69:397-403.

14. Cukras C, Agron E, Klein ML, et al. Natural history of drusenoid pigment epithelial detachment in age-related macular degeneration: Age-Related Eye Disease Study Report No. 28. Ophthalmology. 2010;117:489-499.

15. Taylor HR, West SK. The clinical grading of lens opacities. Aust $N Z$ J Ophthalmol. 1989;17:81-86.

16. Aung KZ, Robman L, Chong EW, English DR, Giles GG, Guymer RH. Non-mydriatic digital macular photography: how good is the second eye photograph? Ophthalmic Epidemiol. 2009; 16:254-261.

17. Scholl HP, Dandekar SS, Peto T, et al. What is lost by digitizing stereoscopic fundus color slides for macular grading in age- 
related maculopathy and degeneration? Ophthalmology. 2004; 111:125-132.

18. Dimitrov PN, Guymer RH, Zele AJ, Anderson AJ, Vingrys AJ. Measuring rod and cone dynamics in age-related maculopathy. Invest Ophthalmol Vis Sci. 2008;49:55-65.

19. Phipps JA, Guymer RH, Vingrys AJ. Loss of cone function in age-related maculopathy. Invest Ophthalmol Vis Sci. 2003;44: 2277-2283.

20. Owsley C, Jackson GR, Cideciyan AV, et al. Psychophysical evidence for rod vulnerability in age-related macular degeneration. Invest Ophthalmol Vis Sci. 2000;41:267-273.

21. Curcio CA, Medeiros NE, Millican CL. Photoreceptor loss in age-related macular degeneration. Invest Ophthalmol Vis Sci. 1996;37:1236-1249.

22. Owsley C, Jackson GR, White M, Feist R, Edwards D. Delays in rod-mediated dark adaptation in early age-related maculopathy. Ophthalmology. 2001;108:1196-1202.

23. Gittings NS, Fozard JL. Age related changes in visual acuity. Exp Gerontol. 1986;21:423-433.

24. Eisner A, Stoumbos VD, Klein ML, Fleming SA. Relations between fundus appearance and function. Eyes whose fellow eye has exudative age-related macular degeneration. Invest Ophthalmol Vis Sci. 1991;32:8-20.

25. Beirne RO, Hogg RE, Stevenson MR, Zlatkova MB, Chakravarthy U, Anderson RS. Severity staging by early features of agerelated maculopathy exhibits weak relationships with functional deficits on SWS grating acuity. Invest Ophthalmol Vis Sci. 2006; 47:4624-4631.

26. Collins M, Brown B. Glare recovery and its relation to other clinical findings in age-related maculopathy. Clin Vision Sci. 1989;4:155-163.

27. Cheng AS, Vingrys AJ. Visual losses in early age-related maculopathy. Optom Vis Sci. 1993;70:89-96.

28. Sunness JS, Massof RW, Bressler NM, Bressler SB. S cone pathway sensitivity in eyes with high risk and low risk drusen characteristics. Appl Opt. 1989;28:1158-1164.

29. Bird, AC. Bruch's membrane change with age. Br J Ophthalmol. 1992;76:166-168.

30. Guymer R, Luthert P, Bird A. Changes in Bruch's membrane and related structures with age. Prog Retin Eye Res. 1999;18: 59-90.
31. Lamb TD, Pugh EN Jr. Dark adaptation and the retinoid cycle of vision. Prog Retin Eye Res. 2004;23:307-380.

32. Pianta M, Kalloniatis M. Characterisation of dark adaptation in human cone pathways: an application of the equivalent background hypothesis. J Physiol. 2000;528:591-608.

33. Formaz F, Riva CE, Geiser M. Diffuse luminance flicker increases retinal vessel diameter in humans. Curr Eye Res. 1997;16:1252-1257.

34. Garhöfer G, Zawinka C, Resch H, Huemer KH, Dorner GT, Schmetterer L. Diffuse luminance flicker increases blood flow in major retinal arteries and veins. Vision Res. 2004;44:833-838.

35. Shakoor A, Blair NP, Mori M, Shahidi M. Chorioretinal vascular oxygen tension changes in response to light flicker. Invest Ophthalmol Vis Sci. 2006;47:4962-4965.

36. Friedman E. Update of the vascular model of AMD. Brit J Ophthalmol. 2004;88:161-163.

37. Friedman E, Krupsky S, Lane AM, et al. Ocular blood flow velocity in age-related macular degeneration. Ophthalmology. 1995; 102:640-646.

38. Chen JC, Fitzke FW, Pauleikhoff D, Bird AC. Functional loss in age-related Bruch's membrane change with choroidal perfusion defect. Invest Ophthalmol Vis Sci. 1992;33:334-340.

39. Pauleikhoff D, Chen JC, Chisholm IH, Bird AC. Choroidal perfusion abnormality with age-related Bruch's membrane change. Am J Ophthalmol. 1990;109:211-217.

40. Ciulla TA, Harris A, Chung HS, et al. Color Doppler imaging discloses reduced ocular blood flow velocities in nonexudative age-related macular degeneration. Am J Ophthalmol. 1999; 128:75-80.

41. Bird AC. Doyne Lecture. Pathogenesis of retinal pigment epithelial detachment in the elderly; the relevance of Bruch's membrane change. Eye (Lond). 1991;5:1-12.

42. Mayer MJ, Spiegler SJ, Ward B, Glucs A, Kim CB. Mid-frequency loss of foveal flicker sensitivity in early stages of age-related maculopathy. Invest Ophthalmol Vis Sci. 1992;33:3136-3142.

43. Phipps JA, Dang TM, Vingrys AJ, Guymer RH. Flicker perimetry losses in age-related macular degeneration. Invest Ophthalmol Vis Sci. 2004;45:3355-3360.

44. Feigl B, Brown B, Lovie-Kitchin J, Swann P. Functional loss in early age-related maculopathy: the ischaemia postreceptoral hypothesis. Eye. 2007;21:689-696. 